University of Wollongong

Research Online

Faculty of Engineering and Information

Faculty of Engineering and Information

Sciences - Papers: Part A

Sciences

$1-1-2010$

\title{
High-energy, kHz-repetition-rate, ps cryogenic Yb:YAG chirped-pulse amplifier
}

Kyung-Han Hong

Massachusetts Institute of Technology

Juliet Gopinath

Massachusetts Institute of Technology

Darren Rand

Massachusetts Institute of Technology

Aleem M. Siddiqui

Massachusetts Institute of Technology

Shu-Wei Huang

Massachusetts Institute of Technology

See next page for additional authors

Follow this and additional works at: https://ro.uow.edu.au/eispapers

Part of the Engineering Commons, and the Science and Technology Studies Commons

Research Online is the open access institutional repository for the University of Wollongong. For further information contact the UOW Library: research-pubs@uow.edu.au 


\title{
High-energy, kHz-repetition-rate, ps cryogenic Yb:YAG chirped-pulse amplifier
}

\begin{abstract}
We demonstrate amplification of picosecond laser pulses to $40 \mathrm{~mJ}$ at a $2 \mathrm{kHz}$ pulse repetition frequency (PRF) from a two-stage cryogenic chirped-pulse Yb:YAG amplifier, composed of a regenerative amplifier (RGA) and a two-pass booster amplifier. The RGA produces $8.2 \mathrm{~mJ}$ of energy at $2 \mathrm{kHz}$ PRF and $13.2 \mathrm{~mJ}$ at $1 \mathrm{kHz}$ PRF with excellent energy stability $(\sim 0.3 \% \mathrm{rms})$ and beam quality ( $\mathrm{M} 2<1.1)$. Pulse stretching and compression are achieved by using a chirped fiber Bragg grating and a multilayer dielectric grating pair, respectively. Compressed 15 ps pulses from the RGA are obtained with a throughput efficiency of $\sim 80 \%$ ( $6.5 \mathrm{~mJ}$ for $2 \mathrm{kHz}$ ). The booster amplifier further amplifies the pulses to $40 \mathrm{~mJ}$ at $2 \mathrm{kHz}$ PRF, and $\sim 32$ $\mathrm{mJ}, \sim 15 \mathrm{ps}$ pulses are expected after compression. The amplifier chain seeded from a femtosecond $\mathrm{Yb}$ fiber laser enables the optical self-synchronization between signal and pump in optical parametric chirped-pulse amplifier applications.
\end{abstract}

\section{Keywords}

rate, repetition, khz, energy, high, cryogenic, pulse, chirped, yag, yb, amplifier, piosecond

Disciplines

Engineering | Science and Technology Studies

\section{Publication Details}

Hong, K., Gopinath, J., Rand, D., Siddiqui, A., Huang, S., Li, E., Eggleton, B., Hybl, J., Yee Fan, T. \& Kartner, F. (2010). High-energy, kHz-repetition-rate, ps cryogenic Yb:YAG chirped-pulse amplifier. Optics Letters, 35 (11), 1752-1754.

\section{Authors}

Kyung-Han Hong, Juliet Gopinath, Darren Rand, Aleem M. Siddiqui, Shu-Wei Huang, Enbang Li, Benjamin J. Eggleton, John D. Hybl, Tso Yee Fan, and Franz X. Kartner 


\title{
High-energy, kHz-repetition-rate, ps cryogenic Yb:YAG chirped-pulse amplifier
}

\author{
Kyung-Han Hong, ${ }^{1, *}$ Juliet T. Gopinath, ${ }^{2}$ Darren Rand, ${ }^{2}$ Aleem M. Siddiqui, ${ }^{1}$ \\ Shu-Wei Huang, ${ }^{1}$ Enbang Li, ${ }^{3}$ Benjamin J. Eggleton, ${ }^{3}$ John D. Hybl, ${ }^{2}$ \\ Tso Yee Fan, ${ }^{2}$ and Franz X. Kärtner ${ }^{1}$ \\ ${ }^{1}$ Department of Electrical Engineering and Computer Science and Research Laboratory of Electronics, \\ Massachusetts Institute of Technology (MIT), Cambridge, Massachusetts 02139, USA \\ ${ }^{2}$ MIT Lincoln Laboratory, Lexington, Massachusetts 02420, USA \\ ${ }^{3}$ Centre for Ultrahigh Bandwidth Devices for Optical Systems, Australian Research Council Centre of Excellence, \\ School of Physics, University of Sydney, Sydney, New South Wales 2006, Australia \\ ${ }^{*}$ Corresponding author: kyunghan@mit.edu
}

Received February 11, 2010; accepted April 19, 2010;

posted April 29, 2010 (Doc. ID 124016); published May 17, 2010

\begin{abstract}
We demonstrate amplification of picosecond laser pulses to $40 \mathrm{~mJ}$ at a $2 \mathrm{kHz}$ pulse repetition frequency (PRF) from a two-stage cryogenic chirped-pulse Yb:YAG amplifier, composed of a regenerative amplifier (RGA) and a two-pass booster amplifier. The RGA produces $8.2 \mathrm{~mJ}$ of energy at $2 \mathrm{kHz}$ PRF and $13.2 \mathrm{~mJ}$ at $1 \mathrm{kHz}$ PRF with excellent energy stability $(\sim 0.3 \% \mathrm{rms})$ and beam quality $\left(M^{2}<1.1\right)$. Pulse stretching and compression are achieved by using a chirped fiber Bragg grating and a multilayer dielectric grating pair, respectively. Compressed 15 ps pulses from the RGA are obtained with a throughput efficiency of $\sim 80 \%(\sim 6.5 \mathrm{~mJ}$ for $2 \mathrm{kHz})$. The booster amplifier further amplifies the pulses to $40 \mathrm{~mJ}$ at $2 \mathrm{kHz}$ PRF, and $\sim 32 \mathrm{~mJ}, \sim 15$ ps pulses are expected after compression. The amplifier chain seeded from a femtosecond $\mathrm{Yb}$-fiber laser enables the optical self-synchronization between signal and pump in optical parametric chirped-pulse amplifier applications. (־) 2010 Optical Society of America

OCIS codes: $140.3280,140.3615,320.7090$.
\end{abstract}

Ultrabroadband high-power optical parametric chirpedpulse amplification (OPCPA) [1] is considered one of the most promising techniques for a driving source in attosecond science because of several interesting capabilities, such as few-cycle-pulse amplification without the need for external nonlinear spectral broadening followed by compression, wavelength selective coverage from the visible to mid-IR, and direct amplification of passively carrier-envelope-phase stabilized mid-IR pulses generated via difference-frequency generation. The ultrabroadband few-cycle OPCPA technique benefits from ps pump lasers, in comparison with ns lasers, because a low stretching factor results in good compressibility, and high-peak-power pumping enables broadband phase matching in short nonlinear media. However, the availability of suitable ps pump sources with good beam quality is still the main challenge for scaling ultrabroadband OPCPA systems. The development of high-energy ps pump sources at $\mathrm{kHz}$ pulse repetition frequency (PRF) is important for energy and power scaling of existing mid-IR few-cycle OPCPA systems for phase-matched high-harmonic generation with high photon energies [2,3]. Multi-mJ ps $\mathrm{Nd}$ :YLF amplifier chains have been developed and used for several OPCPA systems at $1 \mathrm{kHz}$ PRF [4, $\underline{\text { ] }}$, but further energy scaling at $\mathrm{kHz}$ PRF is difficult because of thermal beam distortions [ $[\underline{6}]$ and optical damage issues. Among several laser gain media for ultrashort pulse amplification, the $\mathrm{Yb}$ :YAG crystal is very attractive for scaling both energy and average power due to its suitable emission bandwidth for ps pulse amplification and good thermo-optical properties. The main drawback of the Yb:YAG crystal as a gain medium is its high saturation fluence at room temperature $\left(\sim 9 \mathrm{~J} / \mathrm{cm}^{2}\right)$. This problem has been solved by using thin-disk gain modules or cryogenically cooled bulk crystals. Recently $25 \mathrm{~mJ}$, ps pulses at $3 \mathrm{kHz}$ PRF were demonstrated directly from a thin-disk chirped-pulse ampli- fication (CPA) Yb:YAG regenerative amplifier (RGA) [7] for OPCPA pumping. Meanwhile, based on cryogenic Yb:YAG laser technology [8], a $7.5 \mathrm{~mJ}$, ps RGA at $10 \mathrm{~Hz}$ [9] and a $287 \mathrm{~W}$, ps amplifier at $78 \mathrm{MHz}$ [10] were demonstrated in terms of energy and average power scaling, respectively. Most recently, multipass amplification to $>1 \mathrm{~J}$ of energy at $10 \mathrm{~Hz}$ was reported for application to x-ray laser pumping [11]. In this Letter, we demonstrate a multiten-mJ, ps cryogenic Yb:YAG CPA laser system at $\mathrm{kHz}$ PRF, for the first time to our knowledge. A multi-mJ, ps RGA operating at $1-2 \mathrm{kHz}$ PRF with excellent stability and beam quality is developed, and further amplification to $40 \mathrm{~mJ}$ at $2 \mathrm{kHz}$ is demonstrated in a two-pass amplifier.

The optical layout of the high-power CPA ps cryogenic $\mathrm{Yb}$ :YAG laser system operating at $1-2 \mathrm{kHz} \mathrm{PRF}$ is illustrated in Fig. 1. The CPA chain consists of four subsystems: (a) a ps fiber seed source with a chirped fiber Bragg grating (CFBG) stretcher, (b) multi-mJ kHz cryogenic Yb:YAG RGA, (c) high-energy multipass cryogenic Yb:YAG amplifier, and (d) pulse compressor based on a multilayer dielectric (MLD) grating pair. The front-end oscillator is a homebuilt fs Yb-fiber laser at $78 \mathrm{MHz}(\Delta \lambda=$ $\sim 40 \mathrm{~nm}$ at $1030 \mathrm{~nm}$ ). The output pulses from the $\mathrm{Yb}$-fiber laser are stretched to $\sim 450$ ps by a CFBG with $\mathrm{a} \sim 1 \mathrm{~nm}$ bandwidth centered at $\sim 1029 \mathrm{~nm}$. A homebuilt singlemode Yb-fiber preamplifier in Fig. 1(a) compensates for the power loss in the CFBG caused by bandwidth filtering and coupling losses. An average power of $10 \mathrm{~mW}(0.12 \mathrm{~nJ})$ is available for seeding the cryogenic Yb:YAG RGA. The cw-diode-pumped RGA in Fig. 1(b) is designed for amplification to $\sim 10 \mathrm{~W}$ at $1-2 \mathrm{kHz}$ P $\overline{\mathrm{RF}}$. A $10 \mathrm{~mm}$ long $\mathrm{Yb}: \mathrm{YAG}$ crystal with 2 at.\% doping is pumped by a fiber-coupled (core diameter (diam) of $0.4 \mathrm{~mm}$ ) laser diode (Laserline, $\mathrm{GmbH})$ at $940 \mathrm{~nm}$ and cooled with liquid nitrogen in an evacuated Dewar. The pump beam size at the crystal is $1.2 \mathrm{~mm}$ in diam. The RGA is switched by a $\beta-\mathrm{BaB}_{2} \mathrm{O}_{4}$ 
Pockels cell with a quarter-wave voltage synchronized to the mode-locked pulse train. The output from the RGA is further amplified to tens of $\mathrm{mJ}$ in a multipass amplifier [10], as shown in Fig. 1(c), which can be operated in either a two- or four-pass configuration, as represented by paths (2) and (3), respectively. This amplifier has two $23 \mathrm{~mm}$ long $\mathrm{Yb}$ :YAG crystals with 2 at.\% doping, pumped from both sides by two fiber-coupled (core diam of $0.6 \mathrm{~mm}$ ) diode modules with an available total power of $700 \mathrm{~W}$. The spot size at the crystal at the two-pass configuration is in the range of 2.5-2.8 $\mathrm{mm}$ to keep the amplified fluence at $<1 \mathrm{~J} / \mathrm{cm}^{2}$ for $50 \mathrm{~mJ}$ pulses. The damage threshold of high-energy dielectric mirrors is typically in the range of $\sim 10 \mathrm{~J} / \mathrm{cm}^{2}$ for $10-20 \mathrm{~ns}$ pulses, corresponding to $\sim 1 \mathrm{~J} / \mathrm{cm}^{2}$ for $100-200 \mathrm{ps}$ pulses. The output fluence can be further reduced to $<0.6 \mathrm{~J} / \mathrm{cm}^{2}$ with larger spot sizes (3.3-3.5 mm) for a four-pass scheme-not attempted here. The accumulated $B$ integral is estimated to be $\sim 2 \mathrm{rad}$ at $50 \mathrm{~mJ}$ with $\sim 220 \mathrm{ps}$ pulse duration for both two- and four-pass cases. Finally, the pulses are compressed by an MLD grating pair , as illustrated in Fig. 1(d). These gratings with 1752 lines/mm of groove density and $>95 \%$ of diffraction efficiency at $1030 \mathrm{~nm}$ over $10 \mathrm{~nm}$ of bandwidth can handle an average flux of up to $\sim 1 \mathrm{~kW} / \mathrm{cm}^{2}$ with negligible wavefront distortions. A throughput efficiency of $\sim 80 \%$ for this compressor was measured in the experiment.

The amplification characteristics of the RGA and the compressed pulse duration are shown in Figs. 2(a) and 2(b), respectively. For $2 \mathrm{kHz}$ operation, a maximum average power of $16.4 \mathrm{~W}(8.2 \mathrm{~mJ})$ at $100 \mathrm{~W}$ of pump power was obtained with a slope efficiency of $25 \%$, while for $1 \mathrm{kHz}$ operation, a maximum output energy of $13.2 \mathrm{~mJ}$ at $80 \mathrm{~W}$ of pump power was obtained with a slope efficiency of

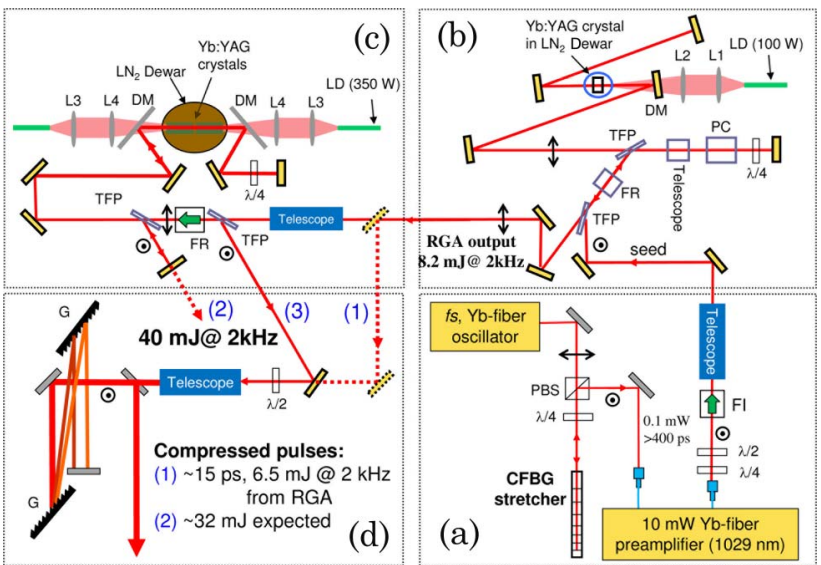

Fig. 1. (Color online) Layout of a high-energy ps laser system at kHz PRF: (a) fiber seed source composed of a Yb-fiber laser, CFBG stretcher, and Yb-fiber preamplifier, (b) $>5 \mathrm{~mJ} \mathrm{kHz}$ cryogenic Yb:YAG RGA, (c) 40 mJ multipass cryogenic Yb:YAG amplifier, and (d) high-energy high-average-power MLD grating compressor. Path (1) represents the direct compression of the RGA output, while paths (2) and (3) show two-pass and four-pass amplification, respectively: PBS, polarization beam splitter; $\lambda / 4$, quarter-wave plate; $\lambda / 2$, half-wave plate; FI/FR, Faraday isolator/rotator; CFBG, chirped fiber Bragg grating; TFP, thin-film polarizer; PC, Pockels cell; L1-L4, lenses; LD, fiber-coupled laser diode; DM, dichroic mirror; G, MLD grating; unspecified mirrors are high reflectors at given angles of incidence.

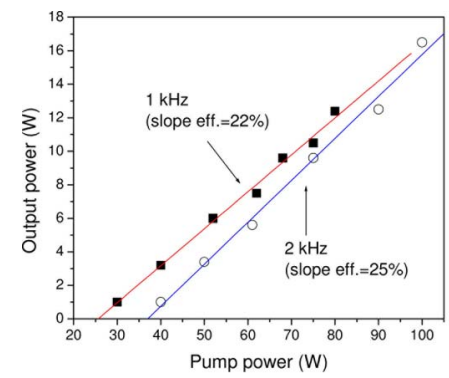

(a)

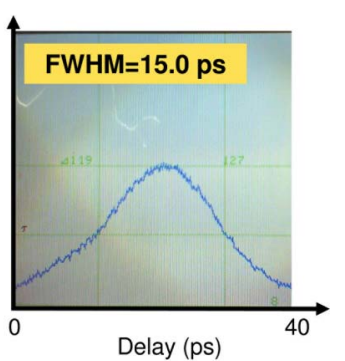

(b)
Fig. 2. (Color online) Amplification of pulses at the kHz RGA and pulse compression: (a) average powers versus pump power at $1 \mathrm{kHz}$ (squares) and $2 \mathrm{kHz}$ (open circles). Slope efficiency is extracted from linear fits and (b) autocorrelation trace of compressed pulses from the RGA. The extracted FWHM pulse duration is 15 ps. Both edges are clipped due to a limited scanning range.

$22 \%$. Because the fluorescence lifetime of the Yb:YAG crystal is $\sim 0.85 \mathrm{~ms}$, the slope efficiency at $1 \mathrm{kHz}(1 \mathrm{~ms}$ interval) is slightly worse than that at $2 \mathrm{kHz}(0.5 \mathrm{~ms}$ interval). However, the total efficiency at $1 \mathrm{kHz}$ for $<80 \mathrm{~W}$ pumping is higher than that at $2 \mathrm{kHz}$ because of a lower amplification threshold that comes from less frequent cavity dumping. At maximum pump power, the RGA is saturated after 10-14 round trips. The shot-to-shot energy stability was as low as $\sim 0.3 \%$ rms for both PRFs. It was reported that the thin-disk kHz RGA in [7] was operated in the regime of "deterministic chaos" regarding PRF due to the low single-pass gain in a thin-disk gain medium and a correspondingly large number of round trips (120-150). In our cryogenic Yb:YAG RGA, no PRF instabilities were observed under normal operating conditions with proper dumping timing and cavity alignment. Grishin et al. [12] showed that RGAs with a relatively low number of round trips $(<15)$ are fundamentally more stable than those with large numbers of round trips, which accounts for the lack of PRF instabilities in our RGA. The gain narrowing of the RGA reduced the spectral bandwidth to $0.24 \mathrm{~nm}$ centered at $1029.2 \mathrm{~nm}$. The pulse duration, taken from path (1) of Fig. 1 and compressed with $\sim 60^{\circ}$ of incidence angle to the first MLD grating and $\sim 1.5 \mathrm{~m}$ of grating separation, was $15 \mathrm{ps}$, as shown in the autocorrelation trace of Fig. 2(b), which is an $\sim 2 \times$ transform-limited pulse duration, 7.5 ps. Because of calibration offsets, the spectral

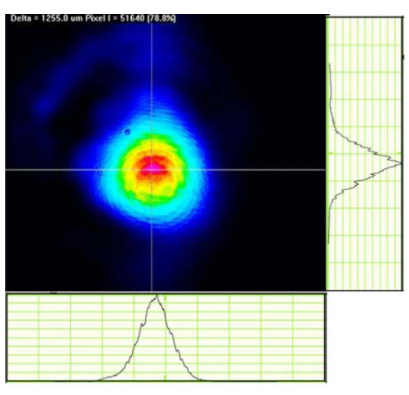

(a)

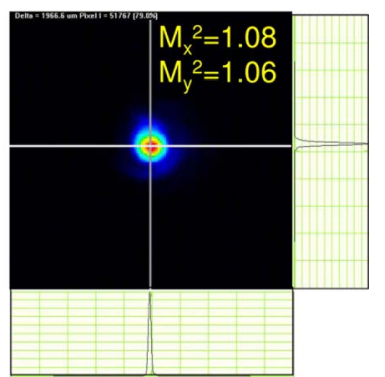

(b)
Fig. 3. (Color online) Spatial beam profile of RGA output at $2 \mathrm{kHz}, 12 \mathrm{~W}$ output power: (a) near-field beam profile with $\sim 2.3 \mathrm{~mm}$ in diam, where the diffraction rings on the main beam come from the measurement setup and (b) far-field (focused) beam profile after compressor. Measured $M^{2}$ values are 1.08 and 1.06 in the horizontal and vertical directions, respectively. 


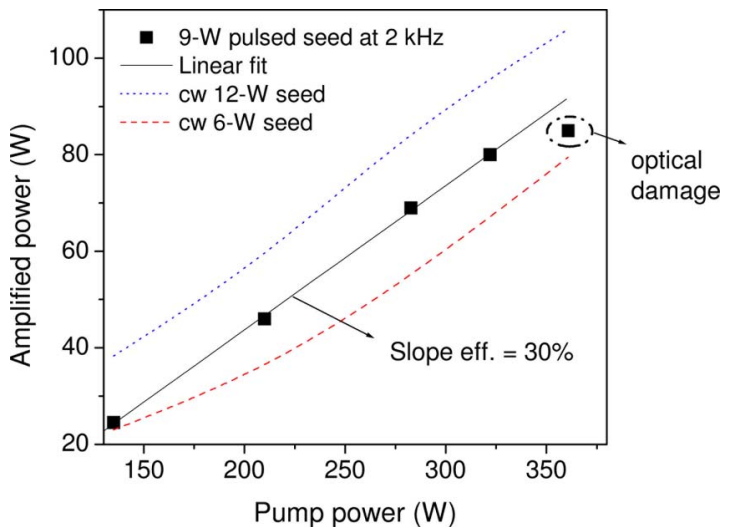

Fig. 4. (Color online) Average power versus pump power in the two-pass amplifier. The slope efficiency is 30\%. Optical damage is observed for output powers at $\sim 85 \mathrm{~W}$. The dotted and dashed curves show the output power for 12 and $6 \mathrm{~W}$ cw seeds for comparison.

window of the CFBG was actually centered at $1028.5 \mathrm{~nm}$, whereas the amplified spectrum from the RGA was at $1029.2 \mathrm{~nm}$, located at the edge of the spectral window of the CFBG. The imperfect compression with the pedestal seems to be attributed mainly to the group-velocity dispersion ripple at this spectral window edge. Thus, the pulse compression can be improved by using a new CFBG centered at $1029.2 \mathrm{~nm}$ or by heating the current CFBG for spectral tuning to a longer wavelength. The near- and farfield beam profiles from the RGA are shown in Figs. 3(a) and 3(b), respectively. At $2 \mathrm{kHz}$ PRF we obtained a clean $\mathrm{TEM}_{00}$ fundamental mode with excellent focusing quality, as indicated by $M^{2}$ of 1.08 and 1.06 for horizontal and vertical directions, respectively, measured using $Z$-scan analysis. The near-diffraction-limited far-field pattern of compressed beams shows that the MLD gratings induce no wavefront distortions, as expected.

For further amplification in the multipass amplifier, we changed to a CFBG with a $2 \times$ larger stretching ratio $(\sim 220 \mathrm{ps}$ for $0.24 \mathrm{~nm}$ at the RGA). A longer stretching ratio increases both the surface and bulk damage thresholds. The spectral width of the RGA output pulses did not change with the new CFBG. The spot size at the Yb:YAG crystal in this amplifier is set to $\sim 2.8 \mathrm{~mm}$ diam for two-pass amplification. Figure 4 shows that a maximum power of $80 \mathrm{~W}(40 \mathrm{~mJ})$ at $2 \mathrm{kHz} \overline{\mathrm{P}} \mathrm{RF}$ is obtained at $9 \mathrm{~W}$ seed power from the RGA with a slope efficiency of $30 \%$ and $\sim 320 \mathrm{~W}$ of pump power. The output power is limited by the damage of the chamber windows at $\sim 85 \mathrm{~W}$. The $\mathrm{cw}$ amplification results using 12 and $6 \mathrm{~W} \mathrm{cw}$ seed powers [dotted and dashed curves in Fig. 4] clearly indicate that the achievable output power is limited only by the damage threshold of amplifier optics. The compression of $40 \mathrm{~mJ}$ pulses is expected to generate $\sim 32 \mathrm{~mJ}, \sim 15 \mathrm{ps}$ pulses. $1 \mathrm{kHz}$ operation was not attempted because the amplifier could be damaged at lower average powers due to excessive pulse energies.

In summary, we demonstrated a ps multi-mJ RGA at 1-2 kHz PRF with excellent beam profile and shot-to-shot energy stability, and we further amplified the energy up to $40 \mathrm{~mJ}$ at $2 \mathrm{kHz}$ PRF. This laser is a promising pump source for multi-mJ few-cycle OPCPA systems at $2.2 \mu \mathrm{m}$ [5] and $800 \mathrm{~nm}$ via frequency doubling. Below-damagethreshold operation of the amplifier with pulse energies $>50 \mathrm{~mJ}$ seems to be feasible with four-pass operation and a larger spot size. Modification of the multipass amplifier toward a larger number of passes [11] and even larger stretching will enable further scaling of energy.

This work was supported by the Air Force Office of Scientific Research (FA9550-06-1-0468 and FA955007-1-0014) through the Defense Advanced Research Projects Agency (DARPA) HRS program. The Lincoln Laboratory portion was sponsored by DARPA under Air Force contract FA8721-05-C-0002. J. T. Gopinath's current affiliation is with the Department of Electrical, Computer and Energy Engineering, University of Colorado, Boulder, USA.

\section{References}

1. A. Dubietis, R. Butkus, and A. Piskarskas, IEEE J. Sel. Top. Quantum Electron. 12, 163 (2006).

2. T. Popmintchev, M. C. Chen, O. Cohen, M. E. Grisham, J. J. Rocca, M. M. Murnane, and H. C. Kapteyn, Opt. Lett. 33, 2128 (2008).

3. E. L. Falcão-Filho, V. M. Gkortsas, Ariel Gordon, and Franz X. Kärtner, Opt. Express 17, 11217 (2009).

4. T. Fuji, N. Ishii, C. Y. Teisset, X. Gu, Th. Metzger, A. Baltuska, N. Forget, D. Kaplan, A. Galvanauskas, and F. Krausz, Opt. Lett. 31, 1103 (2006).

5. J. Moses, S.-W. Huang, K.-H. Hong, O. D. Mücke, E. L. FalcãoFilho, A. Benedick, F. Ö. Ilday, A. Dergachev, J. A. Bolger, B. J. Eggleton, and F. X. Kärtner, Opt. Lett. 34, 1639 (2009).

6. P. Bates, Y. Tang, E. Springate, I. N. Ross, G. H. C. New, R. A. Smith, J. W. G. Tisch, and J. P. Marangos, Central Laser Facility Annual Report 2006-2007, p. 221.

7. T. Metzger, A. Schwarz, C. Y. Teisset, D. Sutter, A. Killi, R. Kienberger, and F. Krausz, Opt. Lett. 34, 2123 (2009).

8. T. Y. Fan, D. J. Ripin, R. L. Aggarwal, J. R. Ochoa, B. Chann, M. Tilleman, and J. Spitzberg, IEEE J. Sel. Top. Quantum Electron. 13, 448 (2007).

9. Y. Akahane, M. Aoyama, K. Ogawa, K. Tsuji, S. Tokita, J. Kawanaka, H. Nishioka, and K. Yamakawa, Opt. Lett. 32, 1899 (2007).

10. K.-H. Hong, A. Siddiqui, J. Moses, J. Gopinath, J. Hybl, F. Ö. Ilday, T. Y. Fan, and F. X. Kärtner, Opt. Lett. 33, 2473 (2008).

11. F. J. Furch, B. A. Reagan, B. M. Luther, A. H. Curtis, S. P. Meehan, and J. J. Rocca, Opt. Lett. 34, 3352 (2009).

12. M. Grishin, V. Gulbinas, and A. Michailovas, Opt. Express 15, 9434 (2007). 